\title{
Numerical treatment of stochastic heroin epidemic model
}

\author{
M. Rafiq ${ }^{1 *}$ (D, Ali Raza², M. Usman lqbal², Zubair Butt², Hafiza Anum Naseem², M. Ali Akram², \\ M. Kamran Butt ${ }^{2}$, Adil Khaliq², Qurat-ul-Ain² and Shamrash Azam²
}

\author{
"Correspondence: \\ m.rafiq@ucp.edu.pk \\ ${ }^{1}$ Faculty of Engineering, University \\ of Central Punjab, Lahore, Pakistan \\ Full list of author information is \\ available at the end of the article
}

\begin{abstract}
We have presented the numerical analysis of a stochastic heroin epidemic model in this paper. The mean of stochastic heroin model is itself a deterministic solution. The effect of reproduction number has also been observed in the stochastic heroin epidemic model. We have developed some stochastic explicit and implicitly driven explicit methods for this model. But stochastic explicit methods have flopped for certain values of parameters. In support, some theorems and graphical illustrations are presented.
\end{abstract}

Keywords: Heroin epidemic model; Stochastic differential equations; Stochastic schemes; Convergence analysis

\section{Introduction}

Humans have known about heroin and euphoria inducing properties for a long time. Heroin is also known as smacks, gag, horse, gear, and brown. Opium poppy is the major cause of drugs. In Neolithic times, the cultivation of these opium poppies was found as evidence. More than six thousand years ago, opium poppies were collected and consumed. In this way opium has remained popular drug for millennia. Opium dens were especially common during the 1800s, and first-time drug morphine was extracted from the opium poppy. Ultimately heroin introduced itself as an opiate drug extracted from morphine [1, 2]. In 1897, the Bayer pharmaceutical company of Germany refined heroin and started selling it as a cure for tuberculosis and addiction of drug morphine. Heroin is an opioid derivative, and an estimated 13.5 million people in the world take opioids. But approximately 9.2 million are heroin users. The average cost of a single dose of $0.1 \mathrm{~g}$ of heroin is $\$ 15$ to $\$ 20$ in the US costing between $\$ 150$ and $\$ 200$ per day for an addict to support their habit. According to the national survey on drug use and health (NSBUH), in 2014 about 430,000 Americans reported using heroin in that year with the greatest increase being by young adults aged 18 to 25 . The number of people using heroin in 2016 was 948,000 , which is nearly double the number of people from two years before [3]. These figures may even be low as it is likely that many heroin users simply do not take part in health service.

The biggest side effect of heroin is its addiction. Body changes and the traumatic mental side effects compel the user to increase the dose. The individuals who have been taking the drugs for quite some time will have high tolerance level hence making it all the more

(c) The Author(s) 2019. This article is distributed under the terms of the Creative Commons Attribution 4.0 International License (http://creativecommons.org/licenses/by/4.0/), which permits unrestricted use, distribution, and reproduction in any medium, provided you give appropriate credit to the original author(s) and the source, provide a link to the Creative Commons license, and indicate if changes were made. 
difficult to quit. If one decides to stop using heroin, the after effects include: extreme craving for the drug, restlessness, muscle, bone pain, diarrhea, and vomiting. These can persist between 48 to 72 hours. It also may take several attempts to get rid of it. Approximately, seventeen million peoples are directly affected by these types of drugs in the form of health problems all over the world.

\section{Literature survey}

In the last decade of the twentieth century various mathematical models were proposed to discuss the epidemic dynamics of heroin model. In 2009, Mulone and Straughan suggested in [4] that the model proposed by White and Comiskey in [5] has stable steady states. Wang, Yang, and Li in 2011 preferred bilinear incidence law over standard incidence in the heroin epidemic model. They proposed that the population is not constant with time in [6]. Samanta extended the model in [5] to non-autonomous epidemic form, which was an improved version of the periodic epidemic model. Here, the population has been treated periodically in [7]. Liu and Zhang worked on time delayed heroin epidemic model, which led to the formulation of a delay differential equation system in [8]. In 2013, Haung and Liu in [9] found that, under specific condition, the delay differential equation model can be converted into an ordinary differential equation model which resembles the renowned SIR epidemic model. Abdurahman, Zhang, and Teng in [10] changed a non-autonomous time delayed heroin epidemic model to an autonomous model. Here the non-standard finite difference pattern was applied to get the discretized heroin epidemic model. In 2015 the authors Fang et al. in [2, 11] formulated age-dependent susceptible and treat-age heroine epidemic models respectively. In 2016 Yang, Li, and Zhang in [12] inquired an age structured heroin model. Non-linear incidence rate was discussed instead of ordinary incidence rate. The time delayed heroin epidemic model was also proposed by the authors in [13]. Ma, Liu, and $\mathrm{Li}$ in [14] discussed various types of bifurcation of heroin epidemic model with non-linear contact rate. Besides, the age structured heroin epidemic model was also proposed in [15]. Wangari and Stone discussed the backward bifurcations and stability in the heroin model, and they considered that heroin users can be rehabilitated quickly in [16]. In 2018, a stochastic heroin epidemic model with bilinear incidence and varying population size was obtained from a deterministic version by Liu, Zhang, and $\mathrm{Li}$ in [17]. Liu, Zhang, and Xing inspected the vanishing and existence of ergodic stationary distribution in a stochastic heroine epidemic model. Stochastic Lyapunov function was developed to discuss the extinction of drug users among the population in [18]. Inspired by the previous work contributions, Zhang and Wang acquired a delayed heroin model in 2019. The authors studied the impact of the time delay on heroin model and concluded that the addicts are not cured quickly, rather they need a time period [19]. Random perturbations were introduced in the deterministic heroin epidemic model by Wei, Yang, and $\mathrm{Li}$ in [20].

Although huge improvements have been made towards the utilization of mathematical methods to model infectious disease, very little efforts have been made to impose this work on heroin epidemic models. In this paper, dynamic disease modeling is extended to the drug-using career. Here the drug users are interpreted as people who directly or indirectly hurt themselves or their families with their drug using habit [21]. The author has made his contributions towards the evolution of mathematical epidemiology. Social issues like drug and alcohol use are termed as epidemics, little work has been carried out towards the 
utilization of mathematical modeling techniques to these difficulties [22-26]. Different models of the transmission of heroin have been studied. The usual quantitative schemes like Euler and Runge-Kutta never maintain dynamical possessions as we have seen in the deterministic modeling. We have also seen that in Euler-Maruyama, stochastic Euler and stochastic Runge-Kutta do not maintain the dynamical possessions in stochastic case. So, from this a question arises and needs to be researched more: Could we develop the random emphatical scheme which maintains all the dynamical possessions [27-29]?

A rule introduced in the deterministic case has been used to start the notion stochastic non-standard finite difference scheme (SNSFD). These regulations were given by Mickens. This is the major point of this paper.

The flow of the paper is based on the following sections:

In Sect. 2, the deterministic heroin epidemic model is described. Section 3 explains the construction way of a stochastic heroin epidemic model and its equilibria. Section 4 explains the stochastic numerical schemes for the stochastic heroin epidemic model and its convergence analysis. In Sect. 5, the conclusion and directions are given.

\section{Deterministic heroin epidemic model}

We consider the heroin epidemic model presented in [5] in this section.

Let at any time $t$, the variables be described as $\mathrm{S}$ (denotes the susceptible group of people), $U_{1}$ (denotes the drug users who are not in treatment), and $U_{2}$ (denotes the drug users who are in treatment). In Fig. 1, the diagram of heroin epidemic model is presented.

The model parameters are described as $\Lambda$ (denotes the individuals entering the susceptible population from general population), $\mu$ (denotes the general population natural decease rate), $\delta_{1}$ (denotes the removal rate due to drug related deaths of users not in treatment and the recovery rate of those who stop using drugs without treatment), $\delta_{2}$ (denotes the removal rate due to drug related deaths of users in treatment and the lucky cure rate that leads to drug-free life accompanied by immunity to drug addiction in the time period of modeling), $\beta_{1}$ (denotes the probability of becoming a drug user), $\mathrm{p}$ (denotes the drug users who get treatment), and $\beta_{3}$ (denotes the probability of a drug user in treatment relapsing to untreated use).

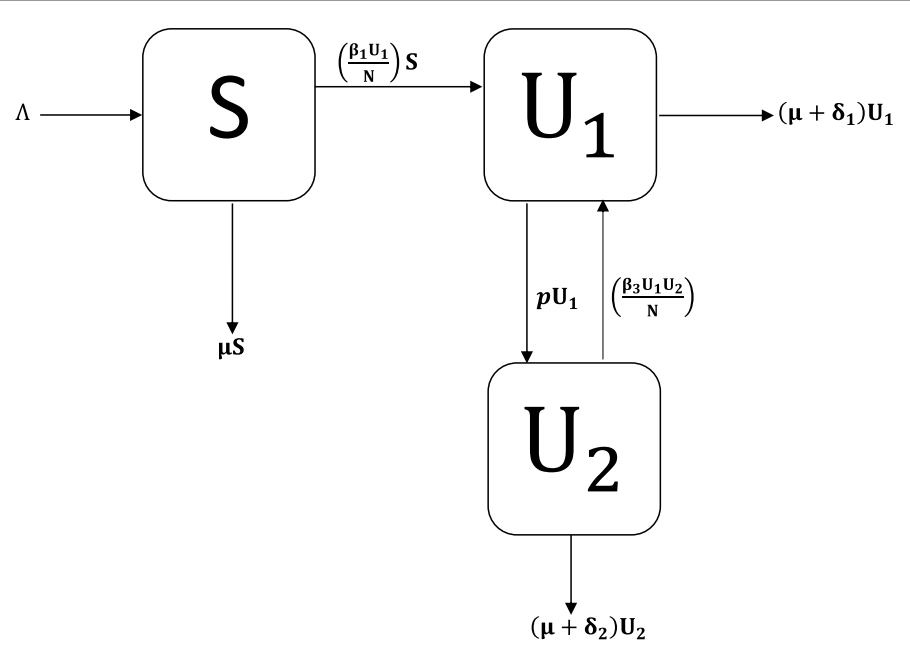

Figure 1 Flow diagram of heroin epidemic model 
The deterministic equations of the heroin epidemic model are as follows:

$$
\begin{aligned}
& \frac{\mathrm{d} \mathrm{S}}{\mathrm{dt}}=\Lambda \mathrm{N}-\frac{\beta_{1} \mathrm{U}_{1} \mathrm{~S}}{\mathrm{~N}}-\mu_{1} \mathrm{~S}, \\
& \frac{\mathrm{dU_{1 }}}{\mathrm{dt}}=\frac{\beta_{1} \mathrm{U}_{1} \mathrm{~S}}{\mathrm{~N}}-\mathrm{p} \mathrm{U}_{1}+\frac{\beta_{3} \mathrm{U}_{1} \mathrm{U}_{2}}{\mathrm{~N}}-\left(\mu+\delta_{1}\right) \mathrm{U}_{1}, \\
& \frac{\mathrm{dU} \mathrm{U}_{2}}{\mathrm{dt}}=\mathrm{p} \mathrm{U}_{1}-\frac{\beta_{3} \mathrm{U}_{1} \mathrm{U}_{2}}{\mathrm{~N}}-\left(\mu+\delta_{2}\right) \mathrm{U}_{2},
\end{aligned}
$$

where

$$
\mathrm{S}+\mathrm{U}_{1}+\mathrm{U}_{2}=\mathrm{N}
$$

put

$$
\mathrm{S}=\frac{\mathrm{S}}{\mathrm{N}}, \quad \mathrm{U}_{1}=\frac{\mathrm{U}_{1}}{\mathrm{~N}}, \quad \mathrm{U}_{2}=\frac{\mathrm{U}_{2}}{\mathrm{~N}}
$$

The normalized form of model (1)-(3) is as follows:

$$
\begin{aligned}
& \frac{\mathrm{d} S}{\mathrm{dt}}=\Lambda-\beta_{1} \mathrm{U}_{1} \mathrm{~S}-\mu_{1} \mathrm{~S}, \\
& \frac{\mathrm{d} \mathrm{U}_{1}}{\mathrm{dt}}=\beta_{1} \mathrm{U}_{1} \mathrm{~S}-\mathrm{p} \mathrm{U}_{1}+\beta_{3} \mathrm{U}_{1} \mathrm{U}_{2}-\mu_{1} \mathrm{U}_{1}, \\
& \frac{\mathrm{d} \mathrm{U}_{2}}{\mathrm{dt}}=\mathrm{pU}_{1}-\beta_{3} \mathrm{U}_{1} \mathrm{U}_{2}-\mu_{1} \mathrm{U}_{2},
\end{aligned}
$$

where the region for system (4)-(6) is $\Gamma=\left\{\left(S, \mathrm{U}_{1}, \mathrm{U}_{2}\right): \mathrm{S}+\mathrm{U}_{1}+\mathrm{U}_{2} \leq \frac{\Lambda}{\mu_{1}}, \mathrm{~S} \geq 0, \mathrm{U}_{1} \geq 0\right.$, $\left.\mathrm{U}_{2} \geq 0\right\}$. The given region is called feasible region for model (4)-(6). So, the solutions of model (4)-(6) lie in this region and are bounded.

\subsection{Equilibria of heroin epidemic model}

The equilibria of heroin model (4)-(6) are as follows:

Drug-free equilibrium is DFE $=\left(\mathrm{S}, \mathrm{U}_{1}, \mathrm{U}_{2}\right)=\left(\frac{\Lambda}{\mu_{1}}, 0,0\right)$,

Drug-present equilibrium is $\mathrm{DPE}=\left(\mathrm{S}^{*}, \mathrm{U}_{1}^{*}, \mathrm{U}_{2}^{*}\right)$, where

$$
\begin{aligned}
\mathrm{U}_{1}^{*}= & \frac{\Lambda-\mu_{1} \mathrm{~S}^{*}}{\beta_{1} \mathrm{~S}^{*}}, \quad \mathrm{U}_{2}^{*}=\frac{\mathrm{p}+\mu_{1}-\beta_{1} \mathrm{~S}^{*}}{\beta_{3}}, \\
\mathrm{~S}^{*}= & {\left[\left(\beta_{1}\left(\mathrm{p}+\mu_{1}\right) \mu_{2}+\mathrm{p} \beta_{3} \mu_{1}-\Lambda \beta_{1} \beta_{3}-\mu_{1}\left(\mathrm{p}+\mu_{1}\right) \beta_{3}\right)\right.} \\
& \pm\left[\left(\Lambda \beta_{1} \beta_{3}+\mu_{1}\left(\mathrm{p}+\mu_{1}\right) \beta_{3}-\beta_{1}\left(\mathrm{p}+\mu_{1}\right) \mu_{2}-\mathrm{p} \beta_{3} \mu_{1}\right)^{2}\right. \\
& \left.\left.-4\left(-\beta_{1}^{2} \mu_{2}-\mu_{1} \beta_{1} \beta_{3}\right)\left(\mathrm{p}+\beta_{3} \Lambda-\beta_{3} \Lambda\left(\mathrm{p}+\mu_{1}\right)\right)\right]^{1 / 2}\right] \\
& /\left[2\left(-\beta_{1}^{2} \mu_{2}-\mu_{1} \beta_{1} \beta_{3}\right)\right], \\
\mathrm{R}_{0}^{\mathrm{d}}= & \frac{\beta_{1}}{\left(\mathrm{p}+\mu_{1}\right)} .
\end{aligned}
$$

Note that $\mathrm{R}_{0}^{\mathrm{d}}$ is heroin generation number. 
Table 1 Transition probabilities

\begin{tabular}{ll}
\hline Transitions $\left(\mathrm{T}_{\mathrm{i}}\right)$ & Probabilities $\left(\mathrm{P}_{\mathrm{i}}\right)$ \\
\hline $\mathrm{T}_{1}=(\Delta \mathrm{W})_{1}=[1,0,0]^{\mathrm{T}}$ & $\mathrm{P}_{1}=\Lambda \Delta \mathrm{t}$ \\
$\mathrm{T}_{2}=(\Delta \mathrm{W})_{2}=[-1,1,0]^{\mathrm{T}}$ & $\mathrm{P}_{2}=\beta_{1} \mathrm{U}_{1} \mathrm{~S} \Delta \mathrm{t}$ \\
$\mathrm{T}_{3}=(\Delta \mathrm{W})_{3}=[-1,0,0]^{\mathrm{T}}$ & $\mathrm{P}_{3}=\mu_{1} \mathrm{~S} \Delta \mathrm{t}$ \\
$\mathrm{T}_{4}=(\Delta \mathrm{W})_{4}=[0,-1,1]^{\mathrm{T}}$ & $\mathrm{P}_{4}=\mathrm{pU}_{1} \Delta \mathrm{t}$ \\
$\mathrm{T}_{5}=(\Delta \mathrm{W})_{5}=[0,1,-1]^{\mathrm{T}}$ & $\mathrm{P}_{5}=\beta_{3} \mathrm{U}_{1} \mathrm{U}_{2} \Delta \mathrm{t}$ \\
$\mathrm{T}_{6}=(\Delta \mathrm{W})_{5}=[0,-1,0]^{\mathrm{T}}$ & $\mathrm{P}_{6}=\mu_{1} \mathrm{U}_{1} \Delta \mathrm{t}$ \\
$\mathrm{T}_{7}=(\Delta \mathrm{W})_{5}=[0,0,-1]^{\mathrm{T}}$ & $\mathrm{P}_{7}=\mu_{2} \mathrm{U}_{2} \Delta \mathrm{t}$ \\
\hline
\end{tabular}

\section{Stochastic heroin epidemic model}

Let us consider the vector $\mathrm{W}=\left[\mathrm{S}, \mathrm{U}_{1}, \mathrm{U}_{2}\right]^{\mathrm{T}}$, the transition probabilities of system (4)-(6) are as follows (see Table 1).

The expectation and variance of stochastic heroin epidemic model is defined as

$$
\begin{aligned}
& \mathrm{E}^{*}[\Delta \mathrm{W}]=\sum_{\mathrm{i}=1}^{7} \mathrm{P}_{\mathrm{i}} \mathrm{T}_{\mathrm{i}}, \\
& \text { Expectation }=\mathrm{E}^{*}[\Delta \mathrm{W}]=\left[\begin{array}{c}
\mathrm{P}_{1}-\mathrm{P}_{2}-\mathrm{P}_{3} \\
\mathrm{P}_{2}-\mathrm{P}_{4}+\mathrm{P}_{5}-\mathrm{P}_{6} \\
\mathrm{P}_{4}-\mathrm{P}_{5}-\mathrm{P}_{7}
\end{array}\right] \Delta \mathrm{t}, \\
& \operatorname{Var}=\mathrm{E}^{*}\left[\Delta \mathrm{W} \Delta \mathrm{W}^{\mathrm{T}}\right]=\sum_{\mathrm{i}=1}^{7} \mathrm{P}_{\mathrm{i}}\left[\mathrm{T}_{\mathrm{i}}\right]\left[\mathrm{T}_{\mathrm{i}}\right]^{\mathrm{T}}, \\
& \mathrm{E}^{*}\left[\Delta \mathrm{W} \Delta \mathrm{W}^{\mathrm{T}}\right]=\left[\begin{array}{ccc}
\mathrm{P}_{1}+\mathrm{P}_{2}+\mathrm{P}_{3} & -\mathrm{P}_{2} & 0 \\
-\mathrm{P}_{2} & \mathrm{P}_{2}+\mathrm{P}_{4+} \mathrm{P}_{5}+\mathrm{P}_{6} & -\mathrm{P}_{4}-\mathrm{P}_{5} \\
0 & -\mathrm{P}_{4}-\mathrm{P}_{5} & \mathrm{P}_{4}+\mathrm{P}_{5}+\mathrm{P}_{7}
\end{array}\right] \Delta \mathrm{t} .
\end{aligned}
$$

The general form of SDEs is as follows:

$$
\begin{aligned}
& \frac{d W(t)}{d t}=f(W(t), t)+L(W(t), t) \frac{d B(t)}{d t}, \\
& \text { Stochastic drift }=f(W(t), t)=\frac{E^{*}[\Delta W]}{\Delta t}, \\
& \text { Stochastic diffusion }=L(W(t), t)=\sqrt{\frac{E^{*}\left[\Delta W \Delta W^{T}\right]}{\Delta t}} .
\end{aligned}
$$

The SDE of system (4)-(6) is as follows:

$$
d W(t)=f(W(t), t) d t+L(W(t), t) d B(t)
$$

with initial conditions $\mathrm{W}(0)=\mathrm{W}_{0}=[0.5,0.3,0.2]^{\mathrm{T}}, 0 \leq \mathrm{t} \leq \mathrm{T}$, and $\mathrm{B}(\mathrm{t})$ is called Brownian motion.

\subsection{Euler-Maruyama method}

System (7) could be written and presented in [30] as follows:

$$
\mathrm{W}_{\mathrm{n}+1}=\mathrm{W}_{\mathrm{n}}+\mathrm{f}\left(\mathrm{W}_{\mathrm{n}}, \mathrm{t}\right) \Delta \mathrm{t}+\mathrm{L}\left(\mathrm{W}_{\mathrm{n}}, \mathrm{t}\right) \Delta \mathrm{B}_{\mathrm{n}},
$$


Table 2 Parameter values [5]

\begin{tabular}{ll}
\hline Parameters & Values (years) \\
\hline$\mu_{1}$ & 0.04 \\
$\Lambda$ & 0.04 \\
$\beta_{1}$ & DFE $=0.02$ \\
& DPE $=0.2$ \\
$\beta_{3}$ & 0.03 \\
$\mathrm{p}$ & 0.02 \\
$\sigma$ & 0.09 \\
\hline
\end{tabular}

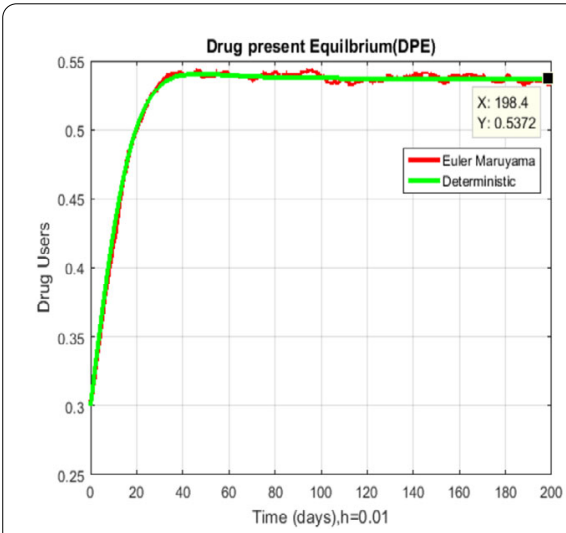

(a)

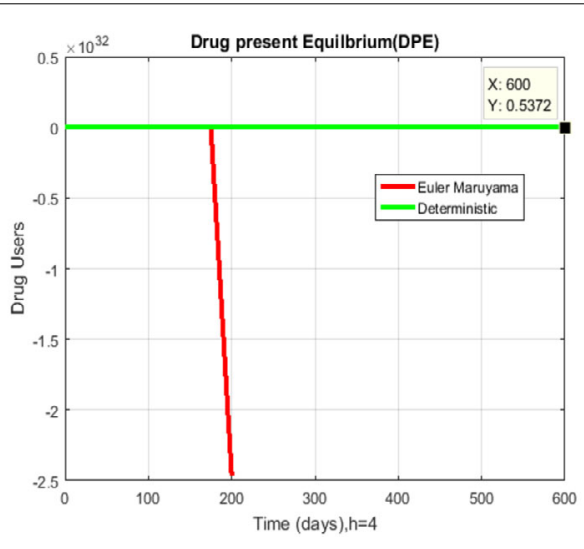

(b)

Figure 2 (a) In drug users' fraction Euler-Maruyama converges to drug-present equilibrium, while deterministic solution is the mean of Euler-Maruyama solution for $\mathbf{h}=0.01$. (b) In drug users' fraction Euler-Maruyama shows negativity and divergence for drug-present equilibrium for $h=4$

where ' $\Delta \mathrm{t}$ ' could be represented as a time step size and $\Delta \mathrm{B}_{\mathrm{n}}$ is the standard normal distribution, i.e., $\Delta B_{n} \sim N(0,1)$. For the solution of system (8), we shall use the parameter values presented in [5] (see Table 2).

The solution of system $(8)$, i.e., $\mathrm{DFE}=\left(\frac{\Lambda}{\mu_{1}}, 0,0\right)$ and DPE $=(0.2713,0.5372,0.1915)$. For the graphical illustration of system (8), see Fig. 2.

\section{Parametric perturbation in heroin epidemic model}

In this technique, we shall choose parameters from system (4)-(6) and change into the random parameters with small noise as $\beta_{1} \mathrm{dt}=\beta_{1} \mathrm{dt}+\sigma \mathrm{dB}$. So, the stochastic heroin epidemic of system (4)-(6) is as follows [31]:

$$
\begin{aligned}
& \mathrm{dS}=\left(\Lambda-\beta_{1} \mathrm{U}_{1} \mathrm{~S}-\mu_{1} \mathrm{~S}\right) \mathrm{dt}-\sigma \mathrm{U}_{1} \mathrm{SdB}, \\
& \mathrm{dU}_{1}=\left(\beta_{1} \mathrm{U}_{1} \mathrm{~S}-\mathrm{pU}_{1}+\beta_{3} \mathrm{U}_{1} \mathrm{U}_{2}-\mu_{1} \mathrm{U}_{1}\right) \mathrm{dt}+\sigma \mathrm{U}_{1} \mathrm{SdB}, \\
& \mathrm{dU}_{2}=\left(\mathrm{pU}_{1}-\beta_{3} \mathrm{U}_{1} \mathrm{U}_{2}-\mu_{2} \mathrm{U}_{2}\right) \mathrm{dt} .
\end{aligned}
$$

The Brownian motion is denoted by $B_{k}(t)(k=1,2,3)$. The stochasticity of system (9)(11) is denoted by $\sigma$ and $\sigma_{1}$. System (9)-(11) has no analytic solution due to non-integral term of Brownian motion. So, in the coming section we shall assume some stochastic methods for the solution of system (9)-(11). 


\subsection{Equilibria of stochastic heroin epidemic model}

The equilibria of system (9)-(11) are as follows:

Drug-free equilibrium is DFE $=\left(S, \mathrm{U}_{1}, \mathrm{U}_{2}\right)=\left(\frac{\Lambda}{\mu_{1}}, 0,0\right)$,

Drug-present equilibrium is DPE $=\left(\mathrm{S}^{*}, \mathrm{U}_{1}^{*}, \mathrm{U}_{2}^{*}\right)$,

where

$$
\begin{aligned}
\mathrm{U}_{1}^{*}= & \frac{\Lambda-\mu_{1} \mathrm{~S}}{\beta_{1} \mathrm{~S}}, \quad \mathrm{U}_{2}^{*}=\frac{\mathrm{p}+\mu_{1}-\beta_{1} \mathrm{~S}}{\beta_{3}}, \\
\mathrm{~S}^{*}= & {\left[\left(\beta_{1}\left(\mathrm{p}+\mu_{1}\right) \mu_{2}+\mathrm{p} \beta_{3} \mu_{1}-\Lambda \beta_{1} \beta_{3}-\mu_{1}\left(\mathrm{p}+\mu_{1}\right) \beta_{3}\right)\right.} \\
& \pm\left[\left(\Lambda \beta_{1} \beta_{3}+\mu_{1}\left(\mathrm{p}+\mu_{1}\right) \beta_{3}-\beta_{1}\left(\mathrm{p}+\mu_{1}\right) \mu_{2}-\mathrm{p} \beta_{3} \mu_{1}\right)^{2}-4\left(-\beta_{1}^{2} \mu_{2}-\mu_{1} \beta_{1} \beta_{3}\right)\right]^{1 / 2} \\
& \left.\times\left(\mathrm{p}+\beta_{3} \Lambda-\beta_{3} \Lambda\left(\mathrm{p}+\mu_{1}\right)\right)\right] \\
& /\left[2\left(-\beta_{1}^{2} \mu_{2}-\mu_{1} \beta_{1} \beta_{3}\right)\right] .
\end{aligned}
$$

Lemma 5.1 The solution $\left(\mathrm{S}(\mathrm{t}), \mathrm{U}_{1}(\mathrm{t}), \mathrm{U}_{2}(\mathrm{t})\right)$ of system (9)-(11), for any assumed initial value $\left(\mathrm{S}(0), \mathrm{U}_{1}(0), \mathrm{U}_{2}(0)\right) \in \mathrm{R}_{+}^{3}$, has the following possessions, almost surely.

\subsubsection{Stochastic threshold dynamics}

Extinction Let us introduce $\mathrm{R}_{0}^{\mathrm{S}}=\mathrm{R}_{0}^{\mathrm{d}}-\frac{\sigma^{2} \mathrm{R}}{2 \mu_{1}^{2}\left(\mathrm{P}+\mu_{1}\right)^{3}}$.

Then we have the following.

Definition 5.1 For system (9)-(11) the drug users $U_{1}(t)$ are supposed to be extinct if $\lim _{\mathrm{t} \rightarrow \infty} \mathrm{U}_{1}(\mathrm{t})=0$ almost surely.

Theorem 5.1 If $\sigma^{2}<\frac{\beta_{1} \mu_{1}}{\Lambda}$ and $\mathrm{R}_{0}^{S}<1$, then the drug users of system (9)-(11) approach to zero exponentially almost surely.

Proof Let us assume that $\left(\mathrm{S}(\mathrm{t}), \mathrm{U}_{1}(\mathrm{t}), \mathrm{U}_{2}(\mathrm{t})\right)$ is a solution of system (9)-(11) with holding the initial conditions $\left(S(0), U_{1}(0), U_{2}(0)\right) \in R_{+}^{3}$ by Ito's formula.

Let $\mathrm{f}\left(\mathrm{U}_{1}\right)=\ln \left(\mathrm{U}_{1}\right)$ and

$$
\begin{aligned}
& \mathrm{d} \ln \left(\mathrm{U}_{1}\right)=\mathrm{f}^{\prime}\left(\mathrm{U}_{1}\right) \mathrm{d} \mathrm{U}_{1}+\frac{1}{2} \mathrm{f}^{\prime \prime}\left(\mathrm{U}_{1}\right) \mathrm{U}_{1}^{2}\left(\sigma^{2} \mathrm{~S}^{2}\right) \mathrm{dt}, \\
& \mathrm{d} \ln \left(\mathrm{U}_{1}\right)=\frac{1}{\mathrm{U}_{1}} \mathrm{~d} \mathrm{U}_{1}+\frac{1}{2}\left(-\frac{1}{\mathrm{U}_{1}^{2}}\right) \mathrm{U}_{1}^{2}\left(\sigma^{2} \mathrm{~S}^{2}\right) \mathrm{dt}, \\
& \mathrm{d} \ln \left(\mathrm{U}_{1}\right)=\left(\beta_{1} S-\mathrm{p}+\beta_{3} \mathrm{U}_{2}-\mu_{1}-\frac{1}{2} \sigma^{2} \mathrm{~S}^{2}\right) \mathrm{dt}+\sigma \mathrm{Sdt} .
\end{aligned}
$$

By integrating " 0 " to " $\mathrm{t}$ " on both sides, we have

$$
\ln \left(\mathrm{U}_{1}\right)=\ln \mathrm{U}_{1}(0)+\int_{0}^{\mathrm{t}}\left(\beta_{1} \mathrm{~S}-\mathrm{p}+\beta_{3} \mathrm{U}_{2}-\mu_{1}-\frac{1}{2} \sigma^{2} \mathrm{~S}^{2}\right) \mathrm{dt}+\int_{0}^{\mathrm{t}} \sigma \mathrm{SdB}
$$

where $\mathrm{M}(\mathrm{t})=\int_{0}^{\mathrm{t}} \sigma \mathrm{SdB}$ is the continuous martingale with $\mathrm{M}(0)=0$. 


$$
\text { If } \begin{aligned}
\sigma^{2}>\frac{\beta_{1} \mu_{1}}{\Lambda} & \\
\ln \left(\mathrm{U}_{1}\right) & \leq\left(\frac{\beta_{1}^{2}}{2 \sigma^{2}}-\left(\mathrm{p}+\mu_{1}\right)\right) \mathrm{t}+\mathrm{M}_{1}(\mathrm{t})+\ln \mathrm{U}_{1}(0), \\
\frac{\ln \left(\mathrm{U}_{1}\right)}{\mathrm{t}} & \leq-\left(\left(\mathrm{p}+\mu_{1}\right)-\frac{\beta_{1}^{2}}{2 \sigma^{2}}\right)+\frac{\mathrm{M}_{1}(\mathrm{t})}{\mathrm{t}}+\frac{\ln \mathrm{U}_{1}(0)}{\mathrm{t}} .
\end{aligned}
$$

By above Lemma 5.1, if

$$
\lim _{t \rightarrow \infty} \frac{M_{1}(t)}{t}=0
$$

and

$$
\lim _{\mathrm{t} \rightarrow \infty} \frac{\ln \mathrm{U}_{1}(\mathrm{t})}{\mathrm{t}} \leq\left(\left(\mathrm{P}+\mu_{1}\right)-\frac{\beta_{1}^{2}}{2 \sigma^{2}}\right)<0,
$$

then $\sigma^{2}>\frac{\beta_{1}^{2}}{2\left(\mathrm{p}+\mu_{1}\right)}$ and $\lim _{\mathrm{t} \rightarrow \infty} \mathrm{U}_{1}(\mathrm{t})=0$ almost surely.

If $\sigma^{2}<\frac{\beta_{1} \mu_{1}}{\Lambda}$, then

$$
\begin{aligned}
& \ln \left(\mathrm{U}_{1}(\mathrm{t})\right) \leq\left(\frac{\beta_{1} \Lambda}{\mu_{1}}-\frac{1}{2} \sigma^{2} \frac{\Lambda^{2}}{\mu_{1}^{2}}-\left(\mathrm{p}+\mu_{1}\right)\right) \mathrm{t}+\mathrm{M}_{1}(\mathrm{t})+\ln \mathrm{U}_{1}(0) \\
& \frac{\ln \mathrm{U}_{1}(\mathrm{t})}{\mathrm{t}} \leq\left(\mathrm{p}+\mu_{1}\right)\left(\frac{\beta_{1} \Lambda}{\mu_{1}\left(\mathrm{p}+\mu_{1}\right)}-\frac{1}{2} \sigma^{2} \frac{\Lambda^{2}}{\mu_{1}^{2}\left(\mathrm{p}+\mu_{1}\right)}-1\right)+\frac{\mathrm{M}_{1}(\mathrm{t})}{\mathrm{t}}+\frac{\ln \mathrm{U}_{1}(0)}{\mathrm{t}} .
\end{aligned}
$$

By taking the superior limit on both sides of (12), we have

$$
\lim _{t \rightarrow \infty} \sup \frac{\ln U_{1}(t)}{t} \leq\left(P+\mu_{1}\right)\left(R_{0}^{S}-1\right),
$$

when $\mathrm{R}_{0}^{\mathrm{S}}<1$ we get

$$
\lim _{t \rightarrow \infty} \sup \frac{\ln U_{1}(t)}{t} \leq 0 \Rightarrow \lim _{t \rightarrow \infty} U_{1}(t)=0
$$

almost surely

$$
\mathrm{R}_{0}^{\mathrm{S}}=\mathrm{R}_{0}^{\mathrm{d}}-\frac{1}{2} \sigma^{2} \frac{\Lambda^{2}}{\mu_{1}^{2}\left(\mathrm{p}+\mu_{1}\right)}<1 .
$$

The stochastic reproduction number $R_{0}^{S}=0.3320<1$ means these measures are helpful in controlling the heroin in population and the stochastic reproduction number $R_{0}^{S}=$ $3.3320>1$ means the heroin is endemic in population.

\subsection{Stochastic Euler method}

System (9)-(11) could be written as follows [31-33]:

$$
\left.\begin{array}{l}
\mathrm{S}^{\mathrm{n}+1}=\mathrm{S}^{\mathrm{n}}+\mathrm{h}\left(\Lambda-\beta_{1} \mathrm{U}_{1}^{\mathrm{n}} \Delta^{\mathrm{n}}-\mu_{1} \Delta^{\mathrm{n}}-\sigma \mathrm{U}_{1}^{\mathrm{n}} \mathrm{S}^{\mathrm{n}} \Delta \mathrm{B}_{\mathrm{n}}\right) \\
\mathrm{U}_{1}^{\mathrm{n}+1}=\mathrm{U}_{1}^{\mathrm{n}}+\mathrm{h}\left(\beta_{1} \mathrm{U}_{1}^{\mathrm{n}} \Delta^{\mathrm{n}}-\mathrm{p} \mathrm{U}_{1}^{\mathrm{n}}+\beta_{3} \mathrm{U}_{1}^{\mathrm{n}} \mathrm{U}_{2}^{\mathrm{n}}-\mu_{1} \mathrm{U}_{1}^{\mathrm{n}}-\sigma \mathrm{U}_{1}^{\mathrm{n}} \mathrm{S}^{\mathrm{n}} \Delta \mathrm{B}_{\mathrm{n}}\right) \\
\mathrm{U}_{2}^{\mathrm{n}+1}=\mathrm{U}_{2}^{\mathrm{n}}+\mathrm{h}\left(\mathrm{p} \mathrm{U}_{1}^{\mathrm{n}}-\beta_{3} \mathrm{U}_{1}^{\mathrm{n}} \mathrm{U}_{2}^{\mathrm{n}}-\mu_{2} \mathrm{U}_{2}^{\mathrm{n}}\right)
\end{array}\right\},
$$




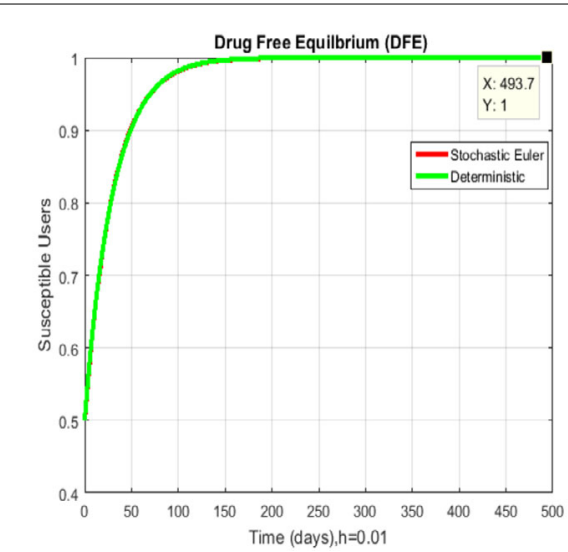

(a)

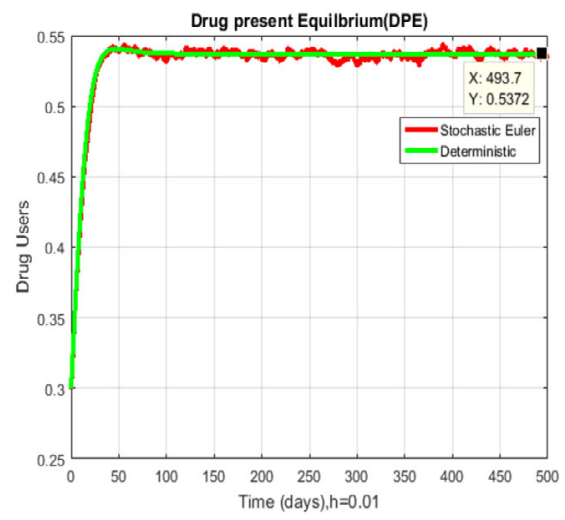

(c)

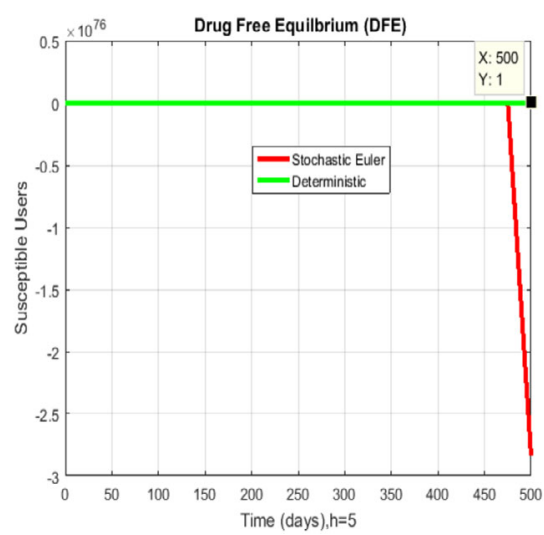

(b)

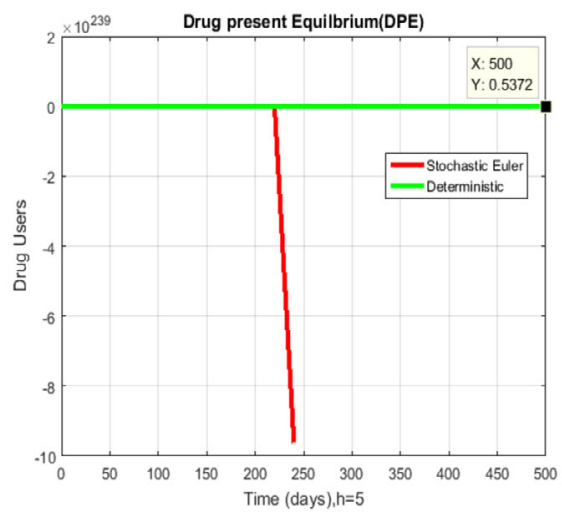

(d)

Figure 3 (a) In susceptible users' fraction stochastic Euler method converges to drug-free equilibrium, while deterministic solution is the mean of stochastic Euler method solution for $h=0.01$. (b) In susceptible users' fraction stochastic Euler method fails to maintain the positivity and diverges for drug-free equilibrium for $h=5$. (c) In drug users' fraction stochastic Euler method converges to drug-present equilibrium, while deterministic solution is the mean of stochastic Euler method solution for $h=0.01$. (d) In drug users' fraction stochastic Euler method shows negativity and unstable behavior for drug-present equilibrium for $h=5$

where " $h$ " is represented as a time step size and $\Delta B_{n} \sim N(0,1)$. The solution of system (13) is illustrated in Fig. 3.

\subsection{Stochastic Runge-Kutta method}

System (9)-(11) could be written as follows [31-33]:

Stage 1

$$
\begin{aligned}
& \mathrm{A}_{1}=\mathrm{h}\left(\Lambda-\beta_{1} \mathrm{U}_{1}^{\mathrm{n}} \mathrm{S}^{\mathrm{n}}-\mu_{1} \mathrm{~S}^{\mathrm{n}}-\sigma \mathrm{U}_{1}^{\mathrm{n}} \mathrm{S}^{\mathrm{n}} \Delta \mathrm{B}_{\mathrm{n}}\right), \\
& \mathrm{B}_{1}=\mathrm{h}\left(\beta_{1} \mathrm{U}_{1}^{\mathrm{n}} S^{\mathrm{n}}-\mathrm{pU}_{1}^{\mathrm{n}}+\beta_{3} \mathrm{U}_{1}^{\mathrm{n}} \mathrm{U}_{2}^{\mathrm{n}}-\mu_{1} \mathrm{U}_{1}^{\mathrm{n}}-\sigma \mathrm{U}_{1}^{\mathrm{n}} \mathrm{S}^{\mathrm{n}} \Delta \mathrm{B}_{\mathrm{n}}\right), \\
& \mathrm{C}_{1}=\mathrm{h}\left(\mathrm{pU}_{1}^{\mathrm{n}}-\beta_{3} \mathrm{U}_{1}^{\mathrm{n}} \mathrm{U}_{2}^{\mathrm{n}}-\mu_{2} \mathrm{U}_{2}^{\mathrm{n}}\right) .
\end{aligned}
$$


Stage 2

$$
\begin{aligned}
\mathrm{A}_{2}= & \mathrm{h}\left(\Lambda-\beta_{1}\left(\mathrm{U}_{1}^{\mathrm{n}}+\frac{\mathrm{B}_{1}}{2}\right)\left(\mathrm{S}^{\mathrm{n}}+\frac{\mathrm{A}_{1}}{2}\right)\right. \\
& \left.-\mu_{1}\left(\mathrm{~S}^{\mathrm{n}}+\frac{\mathrm{A}_{1}}{2}\right)-\sigma\left(\mathrm{U}_{1}^{\mathrm{n}}+\frac{\mathrm{B}_{1}}{2}\right)\left(\mathrm{S}^{\mathrm{n}}+\frac{\mathrm{A}_{1}}{2}\right) \Delta \mathrm{B}_{\mathrm{n}}\right), \\
\mathrm{B}_{2}= & \mathrm{h}\left(\beta_{1}\left(\mathrm{U}_{1}^{\mathrm{n}}+\frac{\mathrm{B}_{1}}{2}\right)\left(\mathrm{S}^{\mathrm{n}}+\frac{\mathrm{A}_{1}}{2}\right)-\mathrm{p}\left(\mathrm{U}_{1}^{\mathrm{n}}+\frac{\mathrm{B}_{1}}{2}\right)+\beta_{3}\left(\mathrm{U}_{1}^{\mathrm{n}}+\frac{\mathrm{B}_{1}}{2}\right)\left(\mathrm{U}_{2}^{\mathrm{n}}+\frac{\mathrm{C}_{1}}{2}\right)\right. \\
& \left.-\mu_{1}\left(\mathrm{U}_{1}^{\mathrm{n}}+\frac{\mathrm{B}_{1}}{2}\right)-\sigma\left(\mathrm{U}_{1}^{\mathrm{n}}+\frac{\mathrm{B}_{1}}{2}\right)\left(\mathrm{S}^{\mathrm{n}}+\frac{\mathrm{A}_{1}}{2}\right) \Delta \mathrm{B}_{\mathrm{n}}\right), \\
\mathrm{C}_{2}= & \mathrm{h}\left[\mathrm{p}\left(\mathrm{U}_{1}^{\mathrm{n}}+\frac{\mathrm{B}_{1}}{2}\right)-\beta_{3}\left(\mathrm{U}_{1}^{\mathrm{n}}+\frac{\mathrm{B}_{1}}{2}\right)\left(\mathrm{U}_{2}^{\mathrm{n}}+\frac{\mathrm{C}_{1}}{2}\right)-\mu_{2}\left(\mathrm{U}_{2}^{\mathrm{n}}+\frac{\mathrm{C}_{1}}{2}\right)\right] .
\end{aligned}
$$

Stage 3

$$
\begin{aligned}
\mathrm{A}_{3}= & \mathrm{h}\left(\Lambda-\beta_{1}\left(\mathrm{U}_{1}^{\mathrm{n}}+\frac{\mathrm{B}_{2}}{2}\right)\left(\mathrm{S}^{\mathrm{n}}+\frac{\mathrm{A}_{2}}{2}\right)\right. \\
& \left.-\mu_{1}\left(\mathrm{~S}^{\mathrm{n}}+\frac{\mathrm{A}_{2}}{2}\right)-\sigma\left(\mathrm{U}_{1}^{\mathrm{n}}+\frac{\mathrm{B}_{2}}{2}\right)\left(\mathrm{S}^{\mathrm{n}}+\frac{\mathrm{A}_{2}}{2}\right) \Delta \mathrm{B}_{\mathrm{n}}\right), \\
\mathrm{B}_{3}= & \mathrm{h}\left(\beta_{1}\left(\mathrm{U}_{1}^{\mathrm{n}}+\frac{\mathrm{B}_{2}}{2}\right)\left(\mathrm{S}^{\mathrm{n}}+\frac{\mathrm{A}_{2}}{2}\right)-\mathrm{p}\left(\mathrm{U}_{1}^{\mathrm{n}}+\frac{\mathrm{B}_{2}}{2}\right)+\beta_{3}\left(\mathrm{U}_{1}^{\mathrm{n}}+\frac{\mathrm{B}_{2}}{2}\right)\left(\mathrm{U}_{2}^{\mathrm{n}}+\frac{\mathrm{C}_{2}}{2}\right)\right. \\
& \left.-\mu_{1}\left(\mathrm{U}_{1}^{\mathrm{n}}+\frac{\mathrm{B}_{2}}{2}\right)-\sigma\left(\mathrm{U}_{1}^{\mathrm{n}}+\frac{\mathrm{B}_{2}}{2}\right)\left(\mathrm{S}^{\mathrm{n}}+\frac{\mathrm{A}_{2}}{2}\right) \Delta \mathrm{B}_{\mathrm{n}}\right), \\
\mathrm{C}_{3}= & \mathrm{h}\left[\mathrm{p}\left(\mathrm{U}_{1}^{\mathrm{n}}+\frac{\mathrm{B}_{2}}{2}\right)-\beta_{3}\left(\mathrm{U}_{1}^{\mathrm{n}}+\frac{\mathrm{B}_{2}}{2}\right)\left(\mathrm{U}_{2}^{\mathrm{n}}+\frac{\mathrm{C}_{2}}{2}\right)-\mu_{2}\left(\mathrm{U}_{2}^{\mathrm{n}}+\frac{\mathrm{C}_{2}}{2}\right)\right] .
\end{aligned}
$$

Stage 4

$$
\begin{aligned}
\mathrm{A}_{4}= & \mathrm{h}\left(\Lambda-\beta_{1}\left(\mathrm{U}_{1}^{\mathrm{n}}+\frac{\mathrm{B}_{3}}{2}\right)\left(\mathrm{S}^{\mathrm{n}}+\frac{\mathrm{A}_{3}}{2}\right)\right. \\
& \left.-\mu_{1}\left(\mathrm{~S}^{\mathrm{n}}+\frac{\mathrm{A}_{3}}{2}\right)-\sigma\left(\mathrm{U}_{1}^{\mathrm{n}}+\frac{\mathrm{B}_{3}}{2}\right)\left(\mathrm{S}^{\mathrm{n}}+\frac{\mathrm{A}_{3}}{2}\right) \Delta \mathrm{B}_{\mathrm{n}}\right), \\
\mathrm{B}_{4}= & \mathrm{h}\left(\beta_{1}\left(\mathrm{U}_{1}^{\mathrm{n}}+\frac{\mathrm{B}_{3}}{2}\right)\left(\mathrm{S}^{\mathrm{n}}+\frac{\mathrm{A}_{3}}{2}\right)-\mathrm{p}\left(\mathrm{U}_{1}^{\mathrm{n}}+\frac{\mathrm{B}_{3}}{2}\right)+\beta_{3}\left(\mathrm{U}_{1}^{\mathrm{n}}+\frac{\mathrm{B}_{3}}{2}\right)\left(\mathrm{U}_{2}^{\mathrm{n}}+\frac{\mathrm{C}_{3}}{2}\right)\right. \\
& \left.-\mu_{1}\left(\mathrm{U}_{1}^{\mathrm{n}}+\frac{\mathrm{B}_{3}}{2}\right)-\sigma\left(\mathrm{U}_{1}^{\mathrm{n}}+\frac{\mathrm{B}_{3}}{2}\right)\left(\mathrm{S}^{\mathrm{n}}+\frac{\mathrm{A}_{3}}{2}\right) \Delta \mathrm{B}_{\mathrm{n}}\right), \\
\mathrm{C}_{4}= & h\left[\mathrm{p}\left(\mathrm{U}_{1}^{\mathrm{n}}+\frac{\mathrm{B}_{3}}{2}\right)-\beta_{3}\left(\mathrm{U}_{1}^{\mathrm{n}}+\frac{\mathrm{B}_{3}}{2}\right)\left(\mathrm{U}_{2}^{\mathrm{n}}+\frac{\mathrm{C}_{3}}{2}\right)-\mu_{2}\left(\mathrm{U}_{2}^{\mathrm{n}}+\frac{\mathrm{C}_{3}}{2}\right)\right] .
\end{aligned}
$$

Final stage

$$
\left.\begin{array}{l}
\mathrm{S}^{\mathrm{n}+1}=\mathrm{S}^{\mathrm{n}}+\frac{1}{6}\left[\mathrm{~A}_{1}+2 \mathrm{~A}_{2}+2 \mathrm{~A}_{3}+\mathrm{A}_{4}\right] \\
\mathrm{U}_{1}^{\mathrm{n}+1}=\mathrm{U}_{1}^{\mathrm{n}}+\frac{1}{6}\left[\mathrm{~B}_{1}+2 \mathrm{~B}_{2}+2 \mathrm{~B}_{3}+\mathrm{B}_{4}\right] \\
\mathrm{U}_{2}^{\mathrm{n}+1}=\mathrm{U}_{2}^{\mathrm{n}}+\frac{1}{6}\left[\mathrm{C}_{1}+2 \mathrm{C}_{2}+2 \mathrm{C}_{3}+\mathrm{C}_{4}\right]
\end{array}\right\},
$$

where " $h$ " is represented as a time step size and $\Delta B_{n} \sim N(0,1)$. The solution of system (14) is shown in Fig. 4. 


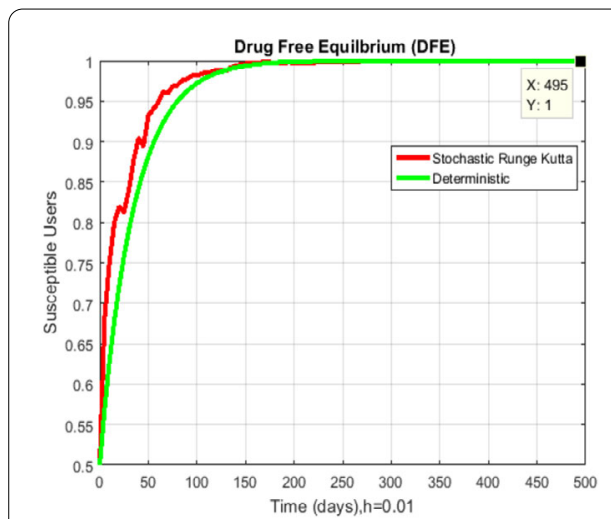

(a)

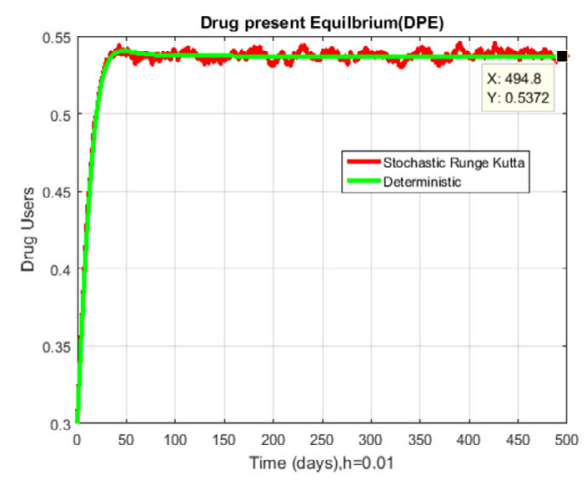

(c)

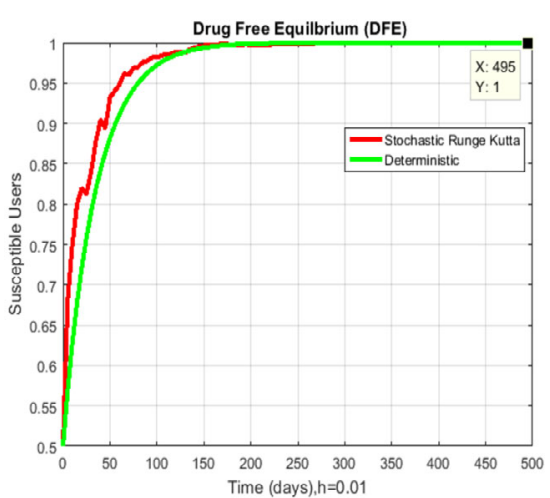

(b)

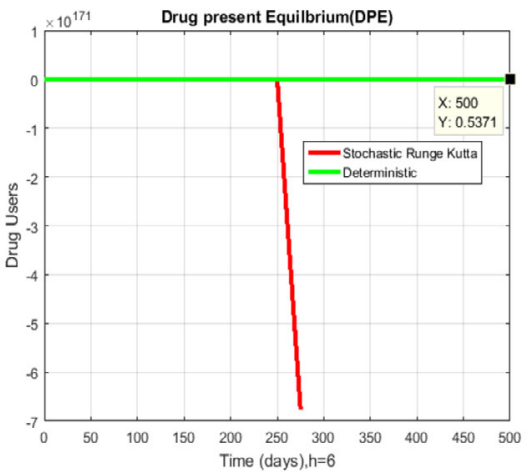

(d)

Figure 4 (a) In susceptible users' fraction stochastic Runge-Kutta method converges to drug-free equilibrium, while deterministic solution is the mean of stochastic Runge-Kutta method solution for $\mathbf{h}=0.01$. (b) In susceptible users' fraction stochastic Runge-Kutta method solution is unbound for drug-free equilibrium for $\mathbf{h}=0.01$. (c) In drug users' fraction stochastic Runge-Kutta method converges to drug-present equilibrium, while deterministic solution is the mean of stochastic Runge-Kutta method solution for $\mathbf{h}=0.01$. (d) In drug users fraction stochastic Runge-Kutta method shows failure of dynamical properties for drug-present equilibrium for $\mathrm{h}=6$

\subsection{Stochastic NSFD method}

System (9)-(11) could be written as follows [31-33]:

$$
\left.\begin{array}{l}
\mathrm{S}^{\mathrm{n}+1}=\frac{\mathrm{S}^{\mathrm{n}}+\lambda \Lambda}{1+\mathrm{h} \beta_{1} \mathrm{U}_{1}^{\mathrm{n}}+\mathrm{h} \mu_{1}+\mathrm{h} \sigma \mathrm{U}_{1}^{\mathrm{n}} \Delta \mathrm{B}_{\mathrm{n}}} \\
\mathrm{U}_{1}^{\mathrm{n}+1}=\frac{\mathrm{U}_{1}^{\mathrm{n}}+\mathrm{h} \beta_{1} \mathrm{U}_{1}^{\mathrm{n}} \mathrm{S}^{\mathrm{n}}+\beta_{3} \mathrm{U}_{1}^{\mathrm{n}} \mathrm{U}_{2}^{\mathrm{n}}+\sigma \mathrm{U}_{1}^{\mathrm{n}} \mathrm{S}^{\mathrm{n}} \Delta \mathrm{B}_{\mathrm{n}}}{1+\mathrm{hp}+\mathrm{h} \mu_{1}} \\
\mathrm{U}_{2}^{\mathrm{n}+1}=\frac{\mathrm{U}_{2}^{\mathrm{n}}+\mathrm{hp} \mathrm{U}_{1}^{\mathrm{n}}}{1+\mathrm{h} \beta_{3} \mathrm{U}_{1}^{\mathrm{n}}+\mathrm{h} \mu_{2}}
\end{array}\right\},
$$

where " $h$ " is represented as a time step size and $\Delta B_{n} \sim N(0,1)$.

\subsubsection{Analysis of the stochastic NSFD method}

Let us assume some theorems as follows.

Theorem 5.2 System (15) has a unique positive solution $\left(\mathrm{S}^{\mathrm{n}}, \mathrm{U}_{1}^{\mathrm{n}}, \mathrm{U}_{2}^{\mathrm{n}}\right) \in \mathrm{R}_{+}^{3}$ on $\mathrm{n} \geq 0$, for any initial system $\left(\mathrm{S}^{\mathrm{n}}(0), \mathrm{U}_{1}^{\mathrm{n}}(0), \mathrm{U}_{2}^{\mathrm{n}}(0)\right) \in \mathrm{R}_{+}^{3}$, almost surely. 
Theorem 5.3 The set $\Omega=\left\{\left(S^{\mathrm{n}}, \mathrm{U}_{1}^{\mathrm{n}}, \mathrm{U}_{2}^{\mathrm{n}}\right) \in \mathrm{R}_{+}^{3}: \mathrm{S}^{\mathrm{n}} \geq 0, \mathrm{U}_{1}^{\mathrm{n}} \geq 0, \mathrm{U}_{2}^{\mathrm{n}} \geq 0, \mathrm{~S}^{\mathrm{n}}+\mathrm{U}_{1}^{\mathrm{n}}+\mathrm{U}_{2}^{\mathrm{n}} \leq \frac{\Lambda}{\mu_{1}}\right\}$ for all $\mathrm{n} \geq 0$ is a non-negative invariant for system (15).

Proof System (15) could be written as follows:

$$
\begin{aligned}
& \frac{\mathrm{S}^{\mathrm{n}+1}-\mathrm{S}^{\mathrm{n}}}{\mathrm{h}}=\Lambda-\beta_{1} \mathrm{U}_{1}^{\mathrm{n}} \mathrm{S}^{\mathrm{n}}-\mu_{1} \mathrm{~S}^{\mathrm{n}}-\sigma \mathrm{U}_{1}^{\mathrm{n}} \mathrm{S}^{\mathrm{n}} \Delta \mathrm{B}_{\mathrm{n}}, \\
& \frac{\mathrm{U}_{1}^{\mathrm{n}+1}-\mathrm{U}_{1}^{\mathrm{n}}}{\mathrm{h}}=\beta_{1} \mathrm{U}_{1}^{\mathrm{n}} \mathrm{S}^{\mathrm{n}}-\mathrm{p} \mathrm{U}_{1}^{\mathrm{n}}+\beta_{3} \mathrm{U}_{1}^{\mathrm{n}} \mathrm{U}_{2}^{\mathrm{n}}-\mu_{1} \mathrm{U}_{1}^{\mathrm{n}}+\sigma \mathrm{U}_{1}^{\mathrm{n}} \mathrm{S}^{\mathrm{n}} \Delta \mathrm{B}_{\mathrm{n}}, \\
& \frac{\mathrm{U}_{2}^{\mathrm{n}+1}-\mathrm{U}_{2}^{\mathrm{n}}}{\mathrm{h}}=\mathrm{p} \mathrm{U}_{1}^{\mathrm{n}}-\beta_{3} \mathrm{U}_{1}^{\mathrm{n}} \mathrm{U}_{2}^{\mathrm{n}}-\mu_{2} \mathrm{U}_{2}^{\mathrm{n}} .
\end{aligned}
$$

So,

$$
\begin{aligned}
& \frac{\left(\mathrm{S}^{\mathrm{n}+1}+\mathrm{U}_{1}^{\mathrm{n}+1}+\mathrm{U}_{2}^{\mathrm{n}+1}\right)-\left(\mathrm{S}^{\mathrm{n}}+\mathrm{U}_{1}^{\mathrm{n}}+\mathrm{U}_{2}^{\mathrm{n}}\right)}{\mathrm{h}}=\Lambda-\mu_{1}\left(\mathrm{~S}^{\mathrm{n}}+\mathrm{U}_{1}^{\mathrm{n}}+\mathrm{U}_{2}^{\mathrm{n}}\right), \\
& \mathrm{S}^{\mathrm{n}+1}+\mathrm{U}_{1}^{\mathrm{n}+1}+\mathrm{U}_{2}^{\mathrm{n}+1}=\left(\mathrm{S}^{\mathrm{n}}+\mathrm{U}_{1}^{\mathrm{n}}+\mathrm{U}_{2}^{\mathrm{n}}\right)+\mathrm{h} \Lambda-\mu_{1}\left(\mathrm{~S}^{\mathrm{n}}+\mathrm{U}_{1}^{\mathrm{n}}+\mathrm{U}_{2}^{\mathrm{n}}\right), \\
& \mathrm{S}^{\mathrm{n}+1}+\mathrm{U}_{1}^{\mathrm{n}+1}+\mathrm{U}_{2}^{\mathrm{n}+1} \leq \frac{\Lambda}{\mu_{1}}+\mathrm{h} \Lambda-\mathrm{h} \mu_{1}\left(\frac{\Lambda}{\mu_{1}}\right), \\
& \mathrm{S}^{\mathrm{n}+1}+\mathrm{U}_{1}^{\mathrm{n}+1}+\mathrm{U}_{2}^{\mathrm{n}+1} \leq \frac{\Lambda}{\mu_{1}},
\end{aligned}
$$

almost surely.

Theorem 5.4 Discrete dynamical system (15) has the same equilibria as those of Continuous dynamical system (9)-(11) for all $\mathrm{n} \geq 0$.

Proof The equilibria of system (9)-(11) are as follows:

DFE, i.e., $\mathrm{D}_{3}=\left(\mathrm{S}^{\mathrm{n}}, \mathrm{U}_{1}^{\mathrm{n}}, \mathrm{U}_{2}^{\mathrm{n}}\right)=\left(\frac{\Lambda}{\mu_{1}}, 0,0\right)$,

DPE, i.e., $E_{3}=\left(S^{n}, U_{1}^{n}, U_{2}^{n}\right)$,

where

$$
\begin{aligned}
\mathrm{U}_{1}^{\mathrm{n}}= & \frac{\Lambda-\mu_{1} \mathrm{~S}^{\mathrm{n}}}{\beta_{1} \mathrm{~S}^{\mathrm{n}}}, \quad \mathrm{U}_{2}^{\mathrm{n}}=\frac{\mathrm{p}+\mu_{1}-\beta_{1} \mathrm{~S}^{\mathrm{n}}}{\beta_{3}}, \\
\mathrm{~S}^{\mathrm{n}}= & {\left[\left(\beta_{1}\left(\mathrm{p}+\mu_{1}\right) \mu_{2}+\mathrm{p} \beta_{3} \mu_{1}-\Lambda \beta_{1} \beta_{3}-\mu_{1}\left(\mathrm{p}+\mu_{1}\right) \beta_{3}\right)\right.} \\
& \pm\left[\left(\Lambda \beta_{1} \beta_{3}+\mu_{1}\left(\mathrm{p}+\mu_{1}\right) \beta_{3}-\beta_{1}\left(\mathrm{p}+\mu_{1}\right) \mu_{2}-\mathrm{p} \beta_{3} \mu_{1}\right)^{2}\right. \\
& \left.\left.-4\left(-\beta_{1}^{2} \mu_{2}-\mu_{1} \beta_{1} \beta_{3}\right)\left(\mathrm{p}+\beta_{3} \Lambda-\beta_{3} \Lambda\left(\mathrm{p}+\mu_{1}\right)\right)\right]^{1 / 2}\right] \\
& /\left[2\left(-\beta_{1}^{2} \mu_{2}-\mu_{1} \beta_{1} \beta_{3}\right)\right],
\end{aligned}
$$

almost surely.

Theorem 5.5 The eigenvalues of discrete dynamical system (15) lie in the unit circle for all $\mathrm{n} \geq 0$. 
Proof Let us consider F, G, and H from system (15) as follows:

$$
\begin{aligned}
& \mathrm{F}=\frac{\mathrm{S}+\mathrm{h} \Lambda}{1+\mathrm{h} \beta_{1} \mathrm{U}_{1}+\mathrm{h} \mu_{1}+\mathrm{h} \sigma \mathrm{U}_{1} \Delta \mathrm{B}_{\mathrm{n}}}, \\
& \mathrm{G}=\frac{\mathrm{U}_{1}+\mathrm{h} \beta_{1} \mathrm{U}_{1} \mathrm{~S}+\beta_{3} \mathrm{U}_{1} \mathrm{U}_{2}+\sigma \mathrm{U}_{1} \mathrm{~S} \Delta \mathrm{B}_{\mathrm{n}}}{1+\mathrm{hp}+\mathrm{h} \mu_{1}}, \\
& \mathrm{H}=\frac{\mathrm{U}_{2}+\mathrm{hpU_{1 }}}{1+\mathrm{h} \beta_{3} \mathrm{U}_{1}+\mathrm{h} \mu_{2}} .
\end{aligned}
$$

The Jacobean matrix is defined as

$$
J=\left[\begin{array}{ccc}
\frac{\partial F}{\partial S} & \frac{\partial F}{\partial U_{1}} & \frac{\partial F}{\partial U_{2}} \\
\frac{\partial G}{\partial S} & \frac{\partial G}{\partial U_{1}} & \frac{\partial G}{\partial U_{2}} \\
\frac{\partial H}{\partial S} & \frac{\partial H}{\partial U_{1}} & \frac{\partial H}{\partial U_{2}}
\end{array}\right],
$$

where

$$
\begin{aligned}
& \frac{\partial \mathrm{F}}{\partial \mathrm{S}}=\frac{1}{1+\mathrm{h} \beta_{1} \mathrm{U}_{1}+\mathrm{h} \mu_{1}+\mathrm{h} \sigma \mathrm{U}_{1} \Delta \mathrm{B}_{\mathrm{n}}}, \\
& \frac{\partial \mathrm{F}}{\partial \mathrm{U}_{1}}=\frac{-(\mathrm{S}+\mathrm{h} \Lambda) \mathrm{h} \beta_{1}}{\left(1+\mathrm{h} \beta_{1} \mathrm{U}_{1}+\mathrm{h} \mu_{1}+\mathrm{h} \sigma \mathrm{U}_{1} \Delta \mathrm{B}_{\mathrm{n}}\right)^{2}}, \quad \frac{\partial \mathrm{F}}{\partial \mathrm{U}_{2}}=0, \\
& \frac{\partial \mathrm{G}}{\partial \mathrm{S}}=\frac{\mathrm{h} \beta_{1} \mathrm{U}_{1}}{1+\mathrm{hp}+\mathrm{h} \mu_{1}}, \quad \frac{\partial \mathrm{G}}{\partial \mathrm{U}_{1}}=\frac{1+\mathrm{h} \beta_{1} \mathrm{~S}+\beta_{3} \mathrm{U}_{2}}{1+\mathrm{hp}+\mathrm{h} \mu_{1}}, \quad \frac{\partial \mathrm{G}}{\partial \mathrm{U}_{2}}=\frac{\beta_{3} \mathrm{U}_{1}}{1+\mathrm{hp}+\mathrm{h} \mu_{1}}, \\
& \frac{\partial \mathrm{H}}{\partial \mathrm{S}}=0, \quad \frac{\partial \mathrm{H}}{\partial \mathrm{U}_{1}}=\frac{\mathrm{hp}}{\mathrm{h} \beta_{3}}, \quad \frac{\partial \mathrm{H}}{\partial \mathrm{U}_{2}}=\frac{1}{1+\mathrm{h} \beta_{3} \mathrm{U}_{1}+\mathrm{h} \mu_{2}} .
\end{aligned}
$$

Now we want to linearize the model about the equilibria of model for drug-free equilibrium $\mathrm{D}_{1}=\left(\mathrm{S}, \mathrm{U}_{1}, \mathrm{U}_{2}\right)=\left(\frac{\Lambda}{\mu_{1}}, 0,0\right)$ and $\mathrm{R}_{0}^{\mathrm{S}}<1$.

The given Jacobean is

$$
\mathrm{J}=\left[\begin{array}{ccc}
\frac{1}{1+\mathrm{h} \mu_{1}} & \frac{-\mathrm{h} \beta_{1}\left(\Lambda+\mu_{1} \mathrm{~h} \Lambda\right)}{\mu_{1}\left(1+\mathrm{h} \mu_{1}\right)^{2}} & 0 \\
0 & \frac{\mu_{1}+\mathrm{h} \beta_{1} \Lambda}{\mu_{1}\left(1+\mathrm{hp}+\mathrm{h} \mu_{1}\right)} & 0 \\
0 & \frac{\mathrm{hp}}{\mathrm{h} \beta_{3}} & \frac{1}{1+\mathrm{h} \mu_{2}}
\end{array}\right] .
$$

The eigenvalues of J are as follows:

$$
\begin{aligned}
& \lambda_{1}=\frac{1}{1+\mathrm{h} \mu_{1}}<1, \quad \lambda_{2}=\frac{\mu_{1}+\mathrm{h} \beta_{1} \Lambda}{\mu_{1}\left(1+\mathrm{hp}+\mathrm{h} \mu_{1}\right)}<1, \quad \mathrm{R}_{0}^{\mathrm{S}}<1, \\
& \lambda_{3}=\frac{1}{1+\mathrm{h} \mu_{2}}<1 .
\end{aligned}
$$

This is to guarantee the fact that all the eigenvalues of Jacobean lie in the unit circle. So, system (15) is LAS around $\mathrm{D}_{1}$. So, the graphical illustration of system (15) is presented in Fig. 5.

\subsection{Contrast section}

Let us assume the comparison among the stochastic methods and the stochastic NSFD method in this section as follows. 


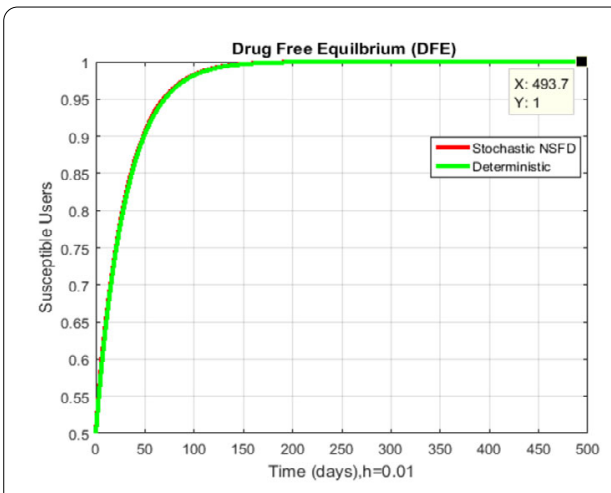

(a)

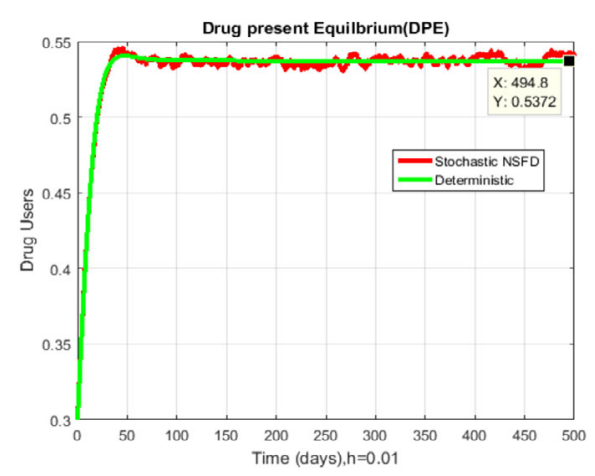

(c)

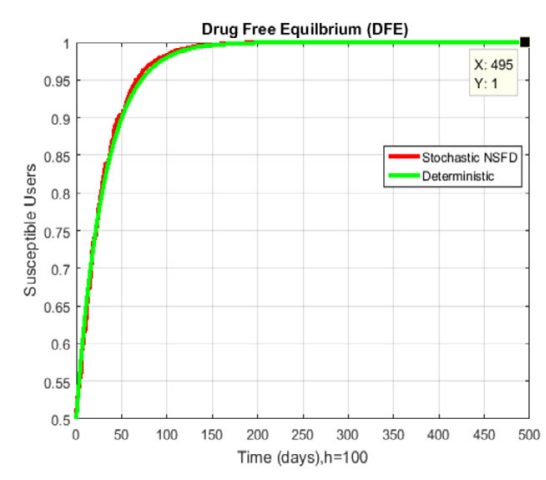

(b)

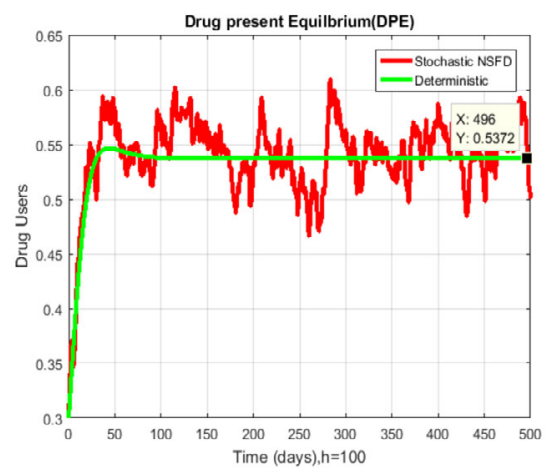

(d)

Figure 5 (a) In susceptible users' fraction stochastic-NSFD converges to drug-free equilibrium, while the deterministic solution is the mean of stochastic NSFD solution for $\mathbf{h}=0.01$. (b) In susceptible users' fraction stochastic-NSFD preserves all dynamical properties for drug-free equilibrium for $\mathbf{h}=100$. (c) In drug users' fraction stochastic-NSFD converges to drug-present equilibrium, while deterministic solution is the mean of stochastic NSFD solution for $\mathbf{h}=0.01$. (d) In drug users' fraction stochastic-NSFD preserves all dynamical properties for drug-present equilibrium, while deterministic solution is the mean of stochastic NSFD solution for $\mathrm{h}=100$

\subsection{Covariance of heroin epidemic model}

Let us describe the covariance among the compartments of heroin epidemic model. For this, we have designed the correlation factors and its consequences as stated in Table 3.

The inverse relationship approved that the number of susceptible users has increased with decrease in the remaining compartments of heroin epidemic model. So, the heroin epidemic model will be a drug-free model.

\section{Results and discussion}

In Fig. 2(a), the given Euler-Maruyama method converges to equilibria of model at $\mathrm{h}=$ 0.01, and unstability could observed in Fig. 2(b). In Fig. 3(a) and 3(c) the stochastic Euler converges to both equilibria at $\mathrm{h}=0.01$. But failure to maintain stability and non-negativity for both equilbria could be observed in Fig. 3(b) and 3(d). In Fig. 4(a) and 4(c), the stochastic Runge-Kutta converges to both equilibria of model at $\mathrm{h}=0.01$, and in Fig. 4(b) and 4(d), the given method shows divergence. So, the stochastic explicit schemes are time dependent and conditionally convergent schemes. On the other hand, in Fig. 5, the stochastic NSFD converges to equilibria of the model for any time. In Fig. 6, we have showed the 


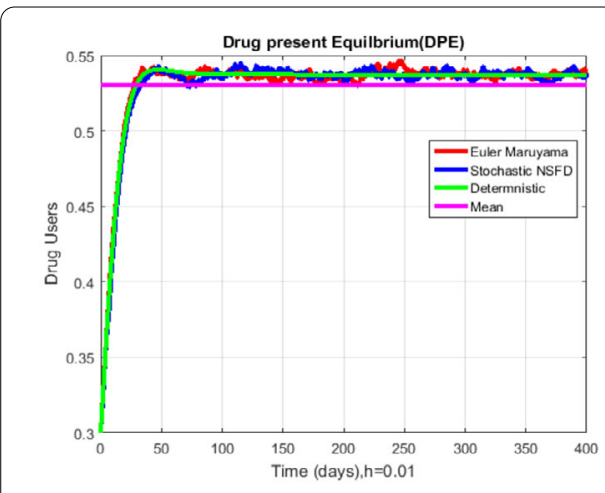

(a)

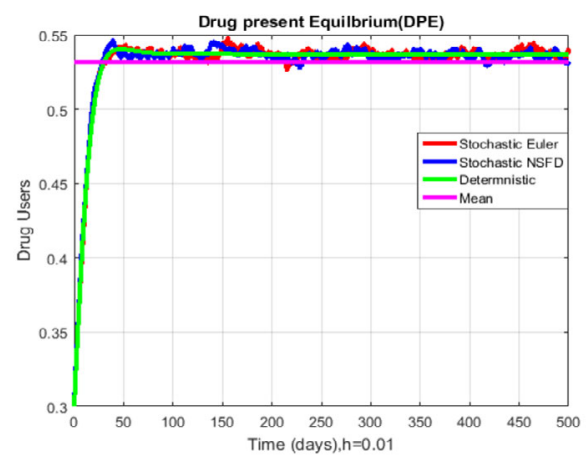

(c)

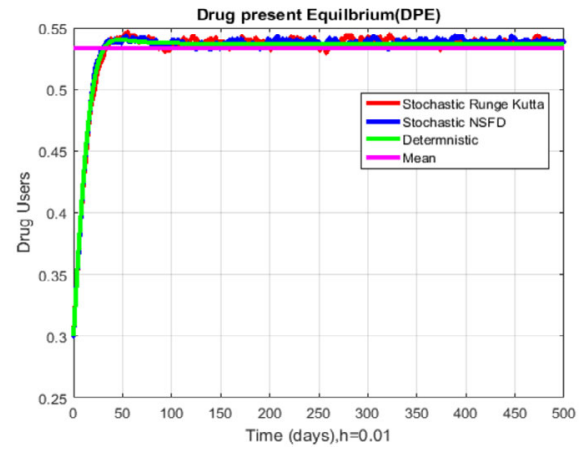

(e )

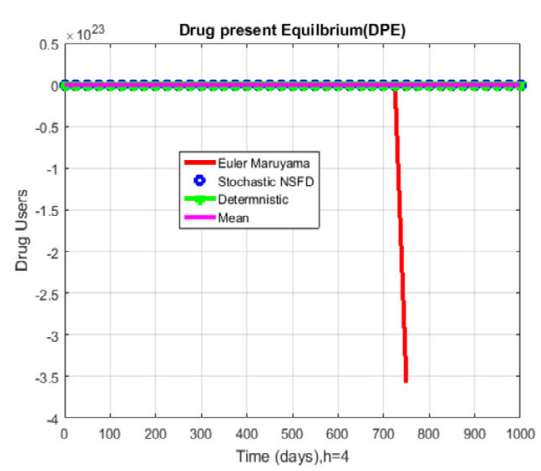

(b)

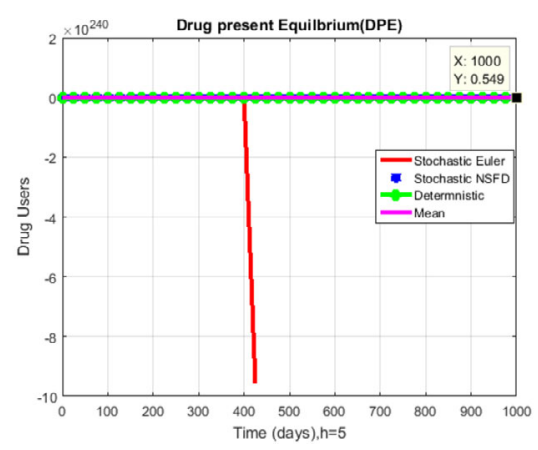

(d)

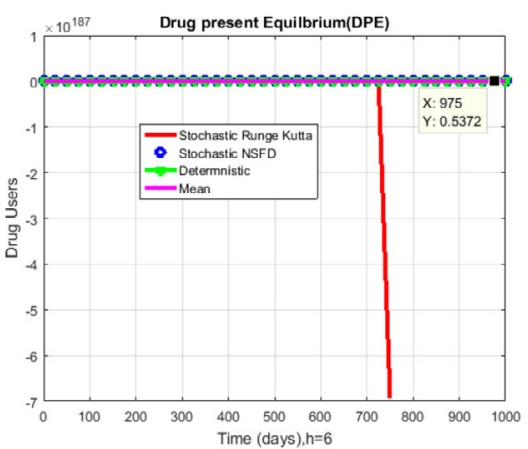

(f)

Figure 6 (a) Drug users' fraction with Euler-Maruyama and its average at $\mathbf{h}=0.01$. (b) Drug users' fraction with Euler-Maruyama and its average at $\mathbf{h}=4$. (c) Drug users' fraction with stochastic Euler and its average at $\mathbf{h}=0.01$. (d) Drug users' fraction with stochastic Euler and its average at $\mathbf{h}=5$. (e) Drug users' fraction with stochastic Runge-Kutta and its average at $\mathbf{h}=0.01$. (f) Drug users' fraction with stochastic Runge-Kutta and its average at $h=6$

efficiency of the stochastic NSFD method with existing stochastic explicit methods for different time step sizes. Also, we can observe in the above graphical illustration that the deterministic solutions are called the average of stochastic solutions of a heroin epidemic system. So, the stochastic explicit methods are time dependent and conditionally convergent methods. This is the beauty of stochastic NSFD as compared to other stochastic explicit methods. 
Table 3 Covariance of model

\begin{tabular}{lll}
\hline Sub-populations & Correlation factor $(\rho)$ & Relationship \\
\hline$\left(\mathrm{S}, \mathrm{U}_{1}\right)$ & -0.8315 & Inverse \\
$\left(\mathrm{U}_{1}, \mathrm{U}_{2}\right)$ & 0.1969 & Direct \\
$\left(\mathrm{S}, \mathrm{U}_{2}\right)$ & -0.4590 & Inverse \\
\hline
\end{tabular}

\section{Conclusion and directions}

In comparison to the deterministic heroin epidemic model, the stochastic heroin epidemic model is a more reliable strategy. The stochastic numerical techniques are detail-oriented, and they work well for even minute time step size. They may lose the necessary properties of a continuous dynamical system due to divergence on specific values of time step size. The SNSFD for a heroin epidemic model is capable of preserving important properties like positivity, dynamical consistency, and boundedness. It is also appropriate for any time step size [27-29]. For our future work, we are aiming to execute SNSFD to sophisticated stochastic delay and spatio-temporal systems. Additionally, we could utilize the current numerical work in the extension of networking flows and fractional networking flows systems [34]. In the future, we are going to work for the reaction diffusion and fractional-order stochastic models.

\section{Acknowledgements}

We would like to thank the referees for their valuable comments.

\section{Funding}

No funding is available for this research project.

\section{Abbreviations}

DFE, Drug-Free Equilibrium; DPE, Drug-Present Equilibrium; NSFD, Non-Standard Finite Difference; ODEs, Ordinary Differential Equations; SDE, Stochastic Differential Equation; SNSFD, Stochastic Non-Standard Finite Difference.

\section{Availability of data and materials}

All data files are available.

Competing interests

We have declared that there are no competing interests.

Authors' contributions

All the authors contributed equally. All authors read and approved the manuscript.

\section{Author details}

${ }^{1}$ Faculty of Engineering, University of Central Punjab, Lahore, Pakistan. ${ }^{2}$ Faculty of Computing, National College of Business Administration and Economics, Lahore, Pakistan.

\section{Publisher's Note}

Springer Nature remains neutral with regard to jurisdictional claims in published maps and institutional affiliations.

Received: 10 July 2019 Accepted: 1 October 2019 Published online: 15 October 2019

\section{References}

1. Davis, G.G.: Complete republication: national association of medical examiners position paper: recommendations for the investigation, diagnosis and certification of deaths related to opioid drugs. J. Med. Toxicol. 10(1), 100-106 (2014)

2. Fang, B., Li, X.Z., Martcheva, M., Cai, L.M.: Global asymptotic properties of a heroin epidemic model with treat-age. Appl. Math. Comput. 263, 315-331 (2015)

3. Evans, W.N., Lieber, E.M., Power, P.: How the reformulation of OxyContin ignited the heroin epidemic. Rev. Econ. Stat. 101(1), 1-15 (2019)

4. Mulone, G., Straughan, B.: A note on heroin epidemics. Math. Biosci. 218(2), 138-141 (2009)

5. White, E., Comiskey, C.: Heroin epidemics, treatment and ODE modelling. Math. Biosci. 208(1), 312-324 (2007)

6. Wang, X., Yang, J., Li, X.: Dynamics of a heroin epidemic model with very population. Appl. Math. 2(6), $732-738$ (2011)

7. Samanta, G.P.: Dynamic behaviour for a nonautonomous heroin epidemic model with time delay. J. Appl. Math. Comput. 35(1-2), 161-178 (2011) 
8. Liu, J., Zhang, T.: Global behaviour of a heroin epidemic model with distributed delays. Appl. Math. Lett. 24(10), 1685-1692 (2011)

9. Huang, G., Liu, A.P.: A note on global stability for a heroin epidemic model with distributed delay. Appl. Math. Lett. 26(7), 687-691 (2013)

10. Abdurahman, X., Zhang, L., Teng, Z.D.: Global dynamics of a discretized heroin epidemic model with time delay. Abstr Appl. Anal. 2014, Article ID 742385 (2014)

11. Fang, B., Li, X.Z., Martcheva, M., Cai, L.M.: Global stability for a heroin model with age-dependent susceptibility. J. Syst. Sci. Complex. 28(6), 1243-1257 (2015)

12. Yang, J., Li, X., Zhang, F.: Global dynamics of a heroin epidemic model with age structure and nonlinear incidence. Int. J. Biomath. 9(3), Article ID 1650033 (2016)

13. Fang, B., Li, X.Z., Martcheva, M., Cai, L.M.: Global stability for a heroin model with two distributed delays. Discrete Contin. Dyn. Syst., Ser. B 19(3), 715-733 (2014)

14. Ma, M.J., Liu, S.Y., Li, J.: Bifurcation of a heroin model with nonlinear incidence rate. Nonlinear Dyn. 88(1), 555-565 (2017)

15. Djilali, S., Touaoula, T.M., Miri, S.E.H.: A heroin epidemic model: very general nonlinear incidence, treat-age and global stability. Acta Appl. Math. 152(1), 171-194 (2017)

16. Wangari, I.M., Stone, L.: Analysis of a heroin epidemic model with saturated treatment function. J. Appl. Math. 2017, Article ID 1953036 (2017)

17. Liu, S., Zhang, L., Zhang, X.B., Li, A.: Dynamics of a stochastic heroin epidemic model with bilinear incidence and varying population size. Int. J. Biomath. 12(5), Article ID 1950005 (2019)

18. Liu, S., Zhang, L., Xing, Y.: Dynamics of a stochastic heroin epidemic model. J. Comput. Appl. Math. 351, 260-269 (2019)

19. Zhang, Z., Wang, Y.: Hopf bifurcation of a heroin model with time delay and saturated treatment function. Adv. Differ. Equ. 2019(1), Article ID 64 (2019)

20. Wei, Y., Yang, Q., Li, G.: Dynamics of the stochastically perturbed heroin epidemic model under non-degenerate noises. Phys. A, Stat. Mech. Appl. 526, Article ID 120914 (2019)

21. Obrien, M., Moran, M.: Overview of Drug Issues in Ireland. Health Research Board, Dublin (1997)

22. Oksendal, B:: Stochastic Differential Equations. Springer, Berlin (2003)

23. Gard, T.C.: Introduction to Stochastic Differential Equations. Dekker, New York (1988)

24. Karatzas, I., Shreve, S.E.: Brownian Motion and Stochastic Calculus, 2nd edn. Springer, Berlin (1991)

25. Karatzas, I., Shreve, S.E.: Brownian Motion and Stochastic Calculus. Springer, Berlin (1988)

26. Allen, E.J., Allen, L.J.S., Arciniega, A., Greenwood, P.E.: Construction of equivalent stochastic differential equation models. Stoch. Anal. Appl. 26(2), 274-297 (2008)

27. Mickens, R.E.: A fundamental principle for constructing nonstandard finite difference schemes for differential equations. J. Differ. Equ. Appl. 11(7), 645-653 (2005)

28. Mickens, R.E.: Nonstandard Finite Difference Models of Differential Equations. World Scientific, Singapore (1994)

29. Mickens, R.E.: Advances in Applications of Nonstandard Finite Difference Schemes. World Scientific, Singapore (1992)

30. Maruyama, G.: Continuous Markov processes and stochastic equations. Rend. Circ. Mat. Palermo 4(1), $48-90$ (1955)

31. Arif, M.S., Raza, A., Rafiq, M., Bibi, M.: A reliable numerical analysis for stochastic hepatitis B virus epidemic model with the migration effect. Iran. J. Sci. Technol., Trans. A, Sci. 43(5), 2477-2492 (2019)

32. Raza, A., Arif, M.S., Rafiq, M.: A reliable numerical analysis for stochastic dengue epidemic model with incubation period of virus. Adv. Differ. Equ. 2019(1), Article ID 32 (2019)

33. Arif, M.S., Raza, A., Rafiq, M., Bibi, M., Fayyaz, R., Naz, M., Javed, U.: A reliable stochastic numerical analysis for typhoid fever incorporating with protection against infection. Comput. Mater. Continua 59(3), 787-804 (2019)

34. Singh, J., Kumar, D., Baleanu, D.: New aspects of fractional Biswas-Milovic model with Mittag-Leffer law. Math. Model. Nat. Phenom. 14(3), Article ID 303 (2019)

\section{Submit your manuscript to a SpringerOpen ${ }^{\circ}$ journal and benefit from:}

- Convenient online submission

- Rigorous peer review

- Open access: articles freely available online

- High visibility within the field

- Retaining the copyright to your article

Submit your next manuscript at $\gg$ springeropen.com 